Article

\title{
Optimization of Emergency Material Dispatch from Multiple Depot Locations to Multiple Disaster Sites
}

\author{
Wei Wang ${ }^{1}$, Li Huang ${ }^{2}$ and Zhaoxia Guo ${ }^{3, *}$ \\ 1 College of Harbor, Coastal and Offshore Engineering, Hohai University, Nanjing 210098, China; \\ 13813826667@163.com \\ 2 School of Public Administration, Hohai University, Nanjing 210098, China; lily8214@126.com \\ 3 Business School, Sichuan University, Chengdu 610065, China \\ * Correspondence: zxguo@scu.edu.cn; Tel.: +86-28-8541-7867
}

Received: 16 October 2017; Accepted: 26 October 2017; Published: 29 October 2017

\begin{abstract}
This study establishes an optimization model of emergency material dispatch with the objectives of the highest reliability and lowest cost when the time frame is uncertain and the emergency material must be dispatched from multiple depot locations to multiple disaster sites. Because the two objectives are not consistent with one another, we propose an algorithm for solving the model by introducing the concept of ideal points, whereby the ideal point between the two objectives is considered the best plan. The study also proves the feasibility and validity of the algorithm with real-life examples.
\end{abstract}

Keywords: emergency material dispatch; reliability; ideal point; multiple disaster sites; multiple depot locations

\section{Introduction}

Sudden disasters and accidents pose a substantial threat to society, safety, and property. Sudden events are characterized by uncertainty, urgency, and a high level of destructiveness and can inflict substantial losses. China is a country with frequent natural disasters. Over the past 10 years, natural disasters, accidents, and public-health and security-related catastrophes have caused more than 200,000 deaths and more than 2 million injuries per year on average. To reduce the casualties and economic loss caused by such emergencies as much as possible, it is important to study the emergency dispatch of relief supplies.

In recent years, researchers have conducted in-depth research on the transportation of emergency materials. For example, Nolz et al. studied the risk of discontinued transportation paths due to damaged infrastructure and addressed such risk as a multi-objective optimization problem [1]. Zheng and Ling proposed a multi-objective fuzzy optimization approach to emergency transportation planning and developed a collaborative optimization method [2]. Yamada viewed the emergency materials dispatch problem in terms of the shortest transport route within a network, which he transformed into an investigation of a network flow algorithm under the condition of road capacity limitation [3]. Huang et al. proposed an assessment method that involved continuous route evaluation while minimizing total transportation time, with the goal of the timely delivery of relief supplies [4].

Several researchers have studied emergency material transportation in combination with location. For instance, focusing on location transportation, Abounacer et al. revealed that the transportation route choice depends on the number and location of disaster relief distribution centers and the needs of disaster victims. They established a multi-objective location transportation model [5]. Ghaffari-Nasab et al. calculated the location-routing problem (LRP) based on probabilistic transport time and used different stochastic programming methods to propose dual-objective mathematical 
programming models. Their aim was to minimize overall system cost while ensuring the principles conformed to minimization of the maximum delivery time [6].

In recent years, research on emergency material dispatch has primarily focused on how multi-objective constrained planning with time- and cost-oriented objectives. Specific research topics include the dispatch of relief supplies and personnel, post-disaster vehicle coordination, and rescue-service distribution and dispatch $[7,8]$. Other researchers have used case studies for qualitative analysis [9]. These studies are based on the assumption of deterministic demand. However, in practice, because of the sudden and unpredictable nature of natural disasters, the demand for relief supplies often exhibits substantial uncertainty. Based on the characteristic of demand uncertainty, researchers have performed a series of studies on the problem of a single depot location and multiple disaster sites under the condition of uncertainty [10,11]. In contrast, in connection with the issue of multiple depot locations and a single disaster site, Liu et al. studied a single target problem, a two-stage problem, and the multi-objective problem of the shortest time and the least number of depot locations [12].

In previous studies, most researchers have addressed the problem of the transport and dispatch of relief supplies using quantitative models. These studies involved various methods, were comprehensive, and achieved useful results. In addition, they promoted the advance of research on emergency material dispatch. However, few studies have focused on the problem of supply dispatch from multiple depot locations to multiple disaster sites under uncertain conditions. For example, extending a robust optimization model, Ben-Tal et al. established an optimal dynamic traffic distribution model based on cell transport to reduce the risk of uncertain demand in the relief supply chain [13]. However, a systematic, in-depth study remains lacking. Thus, this study constructs an optimal emergency material dispatch model from multiple depot locations to multiple disaster sites with multiple objectives of the highest reliability and the lowest cost. An additional variable is time-frame uncertainty. The study introduces the concept of ideal points and proposes an algorithm to solve the problem.

\section{Problem Description}

Consider $n$ rescue stations (indexed by $i$ ), and $m$ disaster sites (indexed by $j$ ). Each rescue station $i(i=1,2, \cdots, n)$ has $a_{1}, a_{2}, \cdots, a_{n}$ stock of a certain supply, each disaster site $j(j=1,2, \cdots, m)$ has $b_{1}, b_{2}, \cdots, b_{m}$ amount of demand for this supply and the supply stock at the rescue stations can satisfy the supply demand at the disaster sites, that is, $\sum_{i=1}^{n} a_{i} \geq \sum_{j=1}^{m} b_{j}$.

The impact of uncertain factors-such as traffic congestion, weather, and road conditions-on the supply dispatch transportation time from rescue station $i$ to disaster site $j$ is uncertain and expressed by the interval number $\widetilde{t}_{i j}=\left[t_{i j}^{1}, t_{i j}^{2}\right]$. Let $t$ denote the emergency time-limit period, $c_{i j}$ the unit transportation cost between rescue station $i$ to disaster site $j, t_{i j}^{1}$ the upper limit value, and $t_{i j}^{2}$ the lower limit value of $\widetilde{t}_{i j}$. The decision objective is to maximize the reliability and minimize the transportation cost for plans with an emergency time frame not exceeding $t$ under the condition that the supply demand is met.

Let $p$ the number of rescue stations. Let $\varphi_{j}$ denote the scheme for disaster site $j$, which represents the set of the amount of emergency resources from emergency depot locations to disaster site $j$. It is defined as a set, $\varphi_{j}=\left\{a_{1 j}, a_{2 j}, \ldots, a_{\mathrm{n} j}\right\}$, where $a_{i j}$ denotes the amount of emergency resource from rescue station $i$ to disaster site $j\left(0<a_{i j} \leq a_{i}\right)$. Let $Z$ denote the set of all feasible schemes, $C F\left(\widetilde{t}_{i j}, t\right)$ the certainty factor of event $\left\{\widetilde{t}_{i j} \leq t\right\}, P\left(\varphi_{j}, t\right)$ the possibility that scheme $\varphi_{j}, \Psi$ the scheme for all disaster sites $\left(\Psi=\bigcup_{j=1}^{m} \varphi_{j}\right)$, and $C(\Psi)$ the cost of scheme $\Psi$. 


\section{Model Construction}

\subsection{Model Assumptions}

The model is based on the following assumptions:

(1) Because of the impact of uncertain factors during transport-such as traffic congestion, weather, and road conditions-the uncertainty of the transportation time is expressed by an interval number.

(2) The unit transport cost from each rescue station to each disaster site is a certain number.

(3) The demand for emergency supplies of all disaster sites at different disaster rating levels is known.

(4) The total quantity of supplies at all rescue stations can meet the needs of all disaster sites.

\subsection{Model Construction}

As the emergency time limit is $t$, introducing a certainty factor and expressing the certainty factor of event $\left\{\widetilde{t}_{i j} \leq t\right\}$ with $C F\left(\widetilde{t}_{i j}, t\right)$ can help avoid plans whose emergency time frame does not exceed $t$. According to the preceding problem description, we define

$$
C F\left(\left\{\widetilde{t}_{i j} \leq t\right\}\right)= \begin{cases}0 & t<t_{i j}^{1} \\ \frac{t-t_{i j}^{1}}{t_{i j}^{2}-t_{i j}^{1}} & t_{i j}^{1} \leq t \leq t_{i j}^{2} \\ 1 & t \geq t_{i j}^{2}\end{cases}
$$

When $t_{i j}^{1}=t_{i j}^{2}, \widetilde{t}_{i j}$ degrades to a real number, and we define

$$
C F\left(\left\{\widetilde{t}_{i j} \leq t\right\}\right)= \begin{cases}0 & t<t_{i j}^{1}=t_{i j}^{2} \\ 1 & t_{i j}^{1}=t_{i j}^{2} \leq t\end{cases}
$$

We use $P\left(\varphi_{j}, t\right)$ to express the possibility that scheme $\varphi_{j}$ (there are $p$ rescue stations in scheme $\varphi_{j}$ ) completes the task of dispatching $b_{j}$ quantity of a certain supply required by disaster site $j$ within emergency time limit period $t$. In addition, $P\left(\varphi_{j}, t\right)=\min C F\left(\underset{\alpha=1,2, \cdots, p}{\cap}\left\{\widetilde{t}_{i_{\alpha} j} \leq t\right\}\right)$, which means that the minimum value of the certainty factor when the time of each rescue station corresponding to the scheme is not greater than $t$ is the probability that the dispatching task can be completed. Using fuzzy reasoning, we have

$$
P\left(\varphi_{j}, t\right)=\min C F\left(\underset{\alpha=1,2, \cdots, p}{\cap}\left\{\widetilde{t}_{i_{\alpha} j} \leq t\right\}\right)=\min _{\alpha=1,2, \cdots, p} C F\left(\left\{\widetilde{t}_{i_{\alpha} j} \leq t\right\}\right)=\min _{\alpha=1,2, \cdots, p} C F\left(\widetilde{t}_{i_{\alpha} j}, t\right)
$$

We use $P(\Psi, t)$ to express the possibility that scheme $\Psi=\bigcup_{j=1}^{m} \varphi_{j}$ completes the task of dispatching a certain supply required by all disaster sites within the emergency time limit period $t$ : $P(\Psi, t)=\min _{j=1,2, \cdots, m} P\left(\varphi_{j}, t\right)$. This study's goal is to determine the scheme with the highest possibility of success among $Z$ on this basis of establishing the lowest rescue cost. With $C(\Psi)$ expressing the cost of scheme $\Psi$, we establish Model (4) as

$$
\begin{aligned}
& \max _{\Psi \subseteq Z}^{P}(\Psi, t) \\
& \min C(\Psi)
\end{aligned}
$$




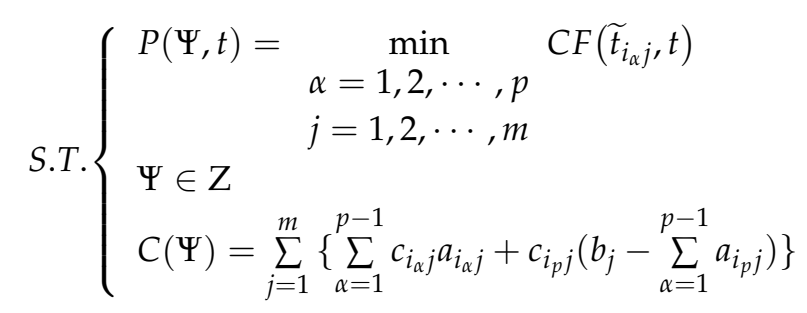

\section{Algorithm for Solving the Model}

The problem is a typical multi-objective programming problem, and this paper uses ideal points to solve it. We obtain the positive ideal points $\bar{P}(\Psi, t)$ and $\bar{C}(\Psi)$ and the negative ideal points $\underline{P}(\Psi, t)$ and $\underline{C}(\Psi)$ for objective functions $P(\Psi, t)$ and $C(\Psi)$. Then, we calculate proximity $\varepsilon$ according to Equation (5) to verify the relative proximity of each feasible scheme to the ideal points. A greater $\varepsilon$ indicates a better solution. At this point, the original multi-objective problem is transformed into a problem of achieving maximum proximity.

$$
\begin{aligned}
& \varepsilon=\frac{R}{R+r}(0 \leq \varepsilon \leq 1) \\
& R=\omega_{1} \frac{P(\Psi, t)}{\bar{P}(\Psi, t)}+\omega_{2} \frac{\bar{C}(\Psi)}{C(\Psi)} \\
& r=\omega_{1} \frac{P(\Psi, t)}{P(\Psi, t)}+\omega_{1} \frac{C(\Psi)}{\underline{C}(\Psi)}
\end{aligned}
$$

where $\omega_{1}$ and $\omega_{2}$ are the weights of the feasible scheme's possibility and the cost of rescue, respectively. Their values are provided by experts, and $\omega_{1}+\omega_{2}=1$.

\subsection{Obtaining Positive and Negative Ideal Points of the Objective Function}

First, we place $C F\left(\widetilde{t}_{i j}, t\right)(i=1,2, \cdots, n)$ into a set of descending sequences ordered from large to small: $C F\left(\widetilde{t}_{i_{1}}, t\right) \geq C F\left(\widetilde{t}_{i_{2}}, t\right) \geq \cdots \geq C F\left(\widetilde{t}_{i_{p}}, t\right) \geq C F\left(\widetilde{t}_{i_{p+1}}, t\right) \geq \cdots \geq C F\left(\widetilde{t}_{i_{n}}, t\right)$. Here, $p$ is the critical subscript index of sequences $a_{i_{1}}, a_{i_{2} j}, \cdots, a_{i_{n} j}$ for demand $b_{j}(j=1,2, \cdots, m)$, and we establish

$$
\begin{gathered}
\varphi_{j}^{\prime}=\left\{a_{i_{1} j}, a_{i_{2} j}, \cdots, b_{j}-\sum_{\alpha=1}^{p-1} a_{i_{\alpha} j}\right\} \\
\Psi^{\prime}=\bigcup_{j=1}^{m} \varphi_{j}^{\prime}
\end{gathered}
$$

At this time, it is most probable that the corresponding emergency demand satisfaction time of scheme $\Psi^{\prime}$ does not exceed the emergency limit time, and the positive ideal point of the objective function $P(\Psi, t)$ is $\bar{P}(\Psi, t)=P\left(\Psi^{\prime}, t\right)=\min _{j=1,2, \cdots, m} C F\left(\widetilde{t}_{i_{p}}, t\right)$. Accordingly, when we place $C F\left(\widetilde{t}_{i j}, t\right)$ $(i=1,2, \cdots, n)$ into a set of ascending sequences ordered from small to large, we obtain the negative ideal point of the objective function as $\underline{P}(\Psi, t)=\min _{j=1,2, \cdots, m} C F\left(\widetilde{t}_{i_{n}}, t\right)$.

Similarly, when sequences $a_{1 j}, a_{2 j}, \cdots, a_{n j}$ are sorted in ascending order based on the size of the $\operatorname{cost} c_{i j}(i=1,2, \cdots, n)$, we obtain a new set of sequences, $a_{c_{1} j}, a_{c_{2} j}, \cdots, a_{c_{n} j}$. Here, $q$ is the critical subscript index of sequences $a_{c_{1} j}, a_{c_{2} j}, \cdots, a_{c_{n} j}$ for demand $b_{j}(j=1,2, \cdots, m)$, and we establish

$$
\begin{gathered}
\varphi_{j}^{\prime \prime}=\left\{a_{c_{1} j}, a_{c_{2} j}, \cdots, b_{j}-\sum_{\alpha=1}^{q-1} a_{\mathcal{c}_{\alpha} j}\right\} \\
\Psi^{\prime \prime}=\bigcup_{j=1}^{m} \varphi_{j}^{\prime \prime}
\end{gathered}
$$

The cost of rescue that corresponds to scheme $\Psi^{\prime \prime}$ is the lowest: $\bar{C}(\Psi)=$ $\sum_{j=1}^{m}\left\{\sum_{\alpha=1}^{q-1} c_{\mathcal{C}_{\alpha} j} a_{\mathcal{c}_{\alpha} j}+c_{\mathcal{c}_{q} j}\left(b_{j}-\sum_{\alpha=1}^{q-1} a_{\mathcal{c}_{\alpha} j}\right)\right\}$. In the same way, when we place sequences $a_{1 j}, a_{2 j}, \cdots, a_{n j}$ into a 
set of descending sequences based on the size of $\operatorname{cost} c_{i j}(i=1,2, \cdots, n)$, we obtain the scheme with the highest cost of rescue and $C(\Psi)$ 's negative ideal point $\underline{C}(\Psi)$.

\subsection{Obtaining Feasible Schemes}

If a scheme shows $P(\Psi, t)=0$, it indicates that the scheme is infeasible. If a scheme shows $P(\Psi, t)>0$, it indicates that the scheme is feasible. If there is no $P(\Psi, t)>0$ for a scheme, it indicates that there is no feasible solution.

\subsection{Algorithm Procedures}

(1) Calculate the positive and negative ideal points.

(2) First, we obtain the certainty factors $C F\left(\widetilde{t}_{i j}, t\right)$ for each rescue station $i$ to each disaster site $j$ according to the definition equation of the certainty factor (1). Next, we place the obtained certainty factors $C F\left(\widetilde{t}_{i j}, t\right)$ into a descending sequence, merge the equivalent items in the sequence, delete the items of number 0 , and obtain sequence $C F(k)(k=1,2, \cdots L)$, with $k=1$.

(3) When establishing $c_{i j}^{\prime}=\left\{\begin{array}{ll}+\infty & C F\left(\widetilde{t}_{i j}, t\right)<C F(k) \\ c_{i j} & C F\left(\widetilde{t}_{i j}, t\right) \geq C F(k)\end{array}\right.$, we obtain that the supply stock at rescue station $i(i=1,2, \cdots, n)$ is $a_{1}, a_{2}, \cdots, a_{n}$ and the supply demand at disaster site $j(j=1,2, \cdots, m)$ is $b_{1}, b_{2}, \cdots, b_{m}$. The transport problem of minimizing unit transport $\operatorname{cost} c_{i j}^{\prime}$ is a classic operations research problem and can be solved with the table operation method. If the problem has solutions, use (4). If the problem has no feasible solutions, use (5).

(4) Calculate the corresponding $P\left(\varphi_{j}, t\right)$ and $C\left(\varphi_{j}\right)$, and use Equation (5) to obtain the corresponding $\varepsilon$ value of each feasible scheme.

(5) When we establish $k=k+1$, the algorithm is terminated if $k>L$. Otherwise, return to (3).

After the preceding calculation, if the problem has no feasible solution, it indicates that the problem has no solution. Otherwise, determine the corresponding $\varepsilon$ value of each feasible scheme. Based on the definition of the ideal point, the scheme with the maximum $\varepsilon$ value is the optimal plan.

\section{Numerical Examples}

We assume there are three disaster sites whose demand for a certain emergency material is $b_{1}=70, b_{2}=80$, and $b_{3}=90$, and nine rescue stations with emergency limit time $t=9$. The time from each rescue station to the disaster sites, the supply stock and the transport cost are shown in Table 1: $C F(k)=(1,0.8,0.75,0.714,0.667,0.6,0.5,0.4)$. We use LINDO 9.0 (LINDO SYSTEMS, Inc, Chicago, USA) to solve the transportation problem. The calculation process and results are shown in Table 2.

Table 1. Known data from each rescue station to the disaster sites.

\begin{tabular}{|c|c|c|c|c|c|c|c|c|c|c|}
\hline \multicolumn{2}{|c|}{ Item } & $A_{1}$ & $A_{2}$ & $A_{3}$ & $A_{4}$ & $A_{5}$ & $A_{6}$ & $A_{7}$ & $A_{8}$ & $A_{9}$ \\
\hline \multirow{3}{*}{$\widetilde{t}_{i j}$} & $B_{1}$ & 3,5 & 8,9 & 5,10 & 5,10 & 6,10 & 4,11 & 5,11 & 7,10 & 6,12 \\
\hline & $B_{2}$ & 5,10 & 6,10 & 4,11 & 5,10 & 7,10 & 7,12 & 3,5 & 2,3 & 5,10 \\
\hline & $B_{3}$ & 7,10 & 6,11 & 3,5 & 2,3 & 8,9 & 5,10 & 5,10 & 4,11 & 5,11 \\
\hline \multicolumn{2}{|c|}{$C F\left(\tilde{t}_{i 1}, t\right)$} & 1 & 1 & 0.8 & 0.8 & 0.75 & 0.714 & 0.667 & 0.667 & 0.5 \\
\hline \multicolumn{2}{|c|}{$C F\left(\widetilde{t}_{i 2}, t\right)$} & 0.8 & 0.75 & 0.714 & 0.667 & 0.667 & 0.4 & 1 & 1 & 0.8 \\
\hline \multicolumn{2}{|c|}{$C F\left(\widetilde{t}_{i 3}, t\right)$} & 0.667 & 0.6 & 1 & 0.8 & 1 & 0.8 & 0.8 & 0.714 & 0.667 \\
\hline \multicolumn{2}{|c|}{$a_{i}$} & 50 & 42 & 40 & 20 & 24 & 14 & 36 & 50 & 46 \\
\hline \multirow{3}{*}{$c_{i j}$} & $B_{1}$ & 9 & 8 & 12 & 6 & 7 & 8 & 4 & 9 & 7 \\
\hline & $B_{2}$ & 6 & 7 & 8 & 4 & 9 & 7 & 9 & 8 & 12 \\
\hline & $B_{3}$ & 4 & 9 & 7 & 9 & 8 & 12 & 6 & 7 & 8 \\
\hline
\end{tabular}


Table 2. Calculation process and results.

\begin{tabular}{|c|c|c|c|c|c|}
\hline$k$ & $C F(k)$ & $j$ & Best Solutions & $P\left(\Psi_{y}, t\right)$ & $C\left(\Psi_{y}\right)$ \\
\hline 1 & 1 & & No solution & & \\
\hline 2 & 0.8 & $\begin{array}{l}j=1 \\
j=2 \\
j=3\end{array}$ & $\begin{array}{c}x_{11}=8, x_{21}=42, x_{31}=20 \\
x_{12}=41, x_{82}=38 \\
x_{33}=40, x_{53}=14, x_{73}=36\end{array}$ & 0.8 & 1692 \\
\hline 3 & 0.75 & $\begin{array}{l}j=1 \\
j=2 \\
j=3\end{array}$ & $\begin{array}{c}x_{21}=40, x_{41}=20, x_{51}=10 \\
x_{12}=50, x_{22}=2, x_{82}=28 \\
x_{33}=40, x_{53}=14, x_{73}=36\end{array}$ & 0.75 & 1656 \\
\hline 4 & 0.714 & $\begin{array}{l}j=1 \\
j=2 \\
j=3\end{array}$ & $\begin{array}{c}x_{21}=12, x_{41}=20, x_{51}=24, x_{61}=14 \\
x_{12}=50, x_{22}=30 \\
x_{33}=4, x_{73}=36, x_{83}=50\end{array}$ & 0.714 & 1600 \\
\hline 5 & 0.667 & $\begin{array}{l}j=1 \\
j=2 \\
j=3\end{array}$ & $\begin{array}{c}x_{51}=24, x_{61}=10, x_{71}=36 \\
x_{22}=42, x_{32}=18, x_{42}=20 \\
x_{13}=50, x_{83}=40\end{array}$ & 0.667 & 1390 \\
\hline 6 & 0.6 & $\begin{array}{l}j=1 \\
j=2 \\
j=3\end{array}$ & $\begin{array}{c}x_{51}=24, x_{61}=10, x_{71}=36 \\
x_{22}=42, x_{32}=18, x_{42}=20 \\
x_{13}=50, x_{83}=40\end{array}$ & 0.6 & 1390 \\
\hline 7 & 0.5 & $\begin{array}{l}j=1 \\
j=2 \\
j=3\end{array}$ & $\begin{array}{c}x_{51}=24, x_{71}=36, x_{91}=10 \\
x_{22}=42, x_{32}=18, x_{42}=20 \\
x_{13}=50, x_{83}=40\end{array}$ & 0.5 & 1380 \\
\hline 8 & 0.4 & $\begin{array}{l}j=1 \\
j=2 \\
j=3\end{array}$ & $\begin{array}{c}x_{51}=24, x_{71}=36, x_{91}=10 \\
x_{22}=42, x_{32}=4, x_{42}=20, x_{62}=14 \\
x_{13}=50, x_{83}=40\end{array}$ & 0.4 & 1366 \\
\hline
\end{tabular}

From Table 2, we can observe that $\bar{P}(\Psi, t)=0.8, \underline{P}(\Psi, t)=0.4, \bar{C}(\Psi)=1366$ and $\underline{C}(\Psi)=2446$. When a disaster occurs, time and reliability are of substantially more importance to emergency rescue than the emergency dispatch cost. Therefore, we assign $\omega_{1}=0.8$ and $\omega_{2}=0.2$, and the calculated $\varepsilon$ of each scheme is $\varepsilon_{2}=0.6411, \varepsilon_{3}=0.6195, \varepsilon_{4}=0.6044, \varepsilon_{5}=0.5927, \varepsilon_{6}=0.5518, \varepsilon_{7}=0.4811$, and $\varepsilon_{8}=0.3969$. Thus, when $k=2$, the corresponding scheme is the optimal one. The specifics of the optimal scheme are as follows: the supplies at rescue stations $A_{1}, A_{2}$, and $A_{3}$ are transported to disaster site $B_{1}$; the supplies at rescue stations $A_{1}$ and $A_{8}$ are transported to disaster site $B_{2}$; and the supplies at rescue stations $A_{3}$, $A_{5}$, and $A_{7}$ are transported to disaster site $B_{3}$.

Numerical results show that a higher reliability level results in a higher emergency dispatch cost. It indicates that improving the reliability of road networks is crucial to emergency rescue. Comparing to the objective of reducing the emergency dispatch cost in emergency rescue, the objectives of reducing the response time and increasing the reliability are more important. In addition, numerical results also show the effectiveness of the proposed algorithm since the algorithm can find the best scheme by only several iterations.

\section{Conclusions}

Taking into account the impact of uncertain factors during the emergency rescue process-such as traffic congestion, weather, and road conditions-this paper uses an interval number to indicate the uncertainty of the transport time and investigates the problem of the emergency dispatch of emergency materials from multiple depot locations to multiple disaster sites under the condition of an uncertain rescue time frame. We establish a multi-objective model that obtains the maximum certainty factor and the minimum transport cost when the emergency time limit does not exceed $t$. In addition, we provide the corresponding solutions and verify the effectiveness of the algorithm with numerical examples. Related research findings include that (1) a higher reliability level led to a higher emergency dispatch cost, which indicates that improving the reliability of road networks is crucial to emergency rescue; 
(2) both time and reliability were much important than the emergency dispatch cost in emergency rescue; and (3) the algorithm proposed was effective since it could find the best scheme by only several iterations.

The paper contributes practical research results and provides solution schemes for the emergency dispatch of relief supplies from multiple depot locations to multiple disaster sites. The algorithm proposed in the study is simple and easy to calculate, and the requisite basic data are easy to obtain. The results can help decision-makers quickly determine a rescue plan after a disaster and thus save valuable rescue time during emergency relief operations.

This research has several limitations as well. This research addressed single-stage emergency resources scheduling. However, multi-stage emergency resources scheduling in a continuous resource consumption process needs to be handled in the real world. This research assumed that the demands of emergency resources were pregiven in all disaster sites. However, it is hard to measure the accurate resources demands of disaster sites due to the uncertainties in emergency rescue. In addition, different types of disasters, roadings, and terrains could have effects on emergency resources scheduling, which have not been investigated in this research. These limitations need to be overcome in the future work. For example, the future work could mathematically handle the grade of roads in the model, as they could affect the time needed from the emergency depot to disaster sites.

Acknowledgments: This work was funded by the National Natural Science Foundation of China (no. 51009060); the Fundamental Research Funds for the Central Universities (no. 2016B09614 and 2017B15614); and Jiangsu Water Conservancy Science and Technology Project (no. 2017059).

Author Contributions: All authors contributed equally to this work. In particular, Wei Wang developed the original idea for the study and designed the methodology. Li Huang drafted the manuscript. Zhaoxia Guo performed the case study and revised the manuscript. All authors have read and approved the final manuscript.

Conflicts of Interest: The authors declare no conflict of interest.

\section{References}

1. Nolz, P.C.; Semet, F.; Doerner, K.F. Risk Approaches for Delivering Disaster Relief Supplies. OR Spectr. 2011, 33, 543-569. [CrossRef]

2. Zheng, Y.J.; Ling, H.F. Emergency Transportation Planning in Disaster Relief Supply Chain Management: A Cooperative Fuzzy Optimization Approach. Soft. Comput. 2013, 17, 1301-1314. [CrossRef]

3. Yamad, T. A network flow approach to a city emergency evacuation planning. Int. J. Syst. Sci. 1996, 27, 931-936. [CrossRef]

4. Huang, M.; Smiloeitz, K.R.; Balcik, B.A. Continuous Approximation Approach for Assessment Routing in Disaster Relief. Transp. Res. B Meth. 2013, 50, 20-41. [CrossRef]

5. Abounacer, R.; Rekik, M.; Renaud, J. An Exact Solution Approach for Multi-objective Location-transportation Problem for Disaster Response. Comput. Oper. Res. 2014, 41, 83-93. [CrossRef]

6. Ghaffarri-Nasab, N.; Jabalameli, M.S.; Aryanezhad, M.B. Modeling and Solving the Bi-Objective Capacitated Location-Routing Problem with Probabilistic Travel Times. Int. J. Adv. Manuf. Technol. 2013, 67, 2007-2019. [CrossRef]

7. Sheu, J.B. An Emergency Logistic Distribution Approach for Quick Response to Urgent Relief Demand in Disasters. Transp. Res. E Logist. Transport. Rev. 2007, 43, 687-709. [CrossRef]

8. Ji, G.; Zhu, C. A Study on Emergency Supply Chain and Risk Based on Urgent Relief Service in Disasters. Syst. Eng. Proced. 2012, 5, 313-325. [CrossRef]

9. Wex, F.; Schryen, G.; Feuerriegel, S. Emergency Response in Natural Disaster Management: Allocation and Scheduling of Rescue Units. Eur. J. Oper. Res. 2014, 235, 697-708. [CrossRef]

10. Nadizadeh, A.; Nasab, H.H. Solving the Dynamic Capacitated Location-Routing Problem with Fuzzy Demands by Hybrid Heuristic Algorithm. Eur. J. Oper. Res. 2014, 238, 458-470. [CrossRef]

11. Wang, H.; Du, L.; Ma, S. Multi-Objective Open Location-Routing Model with Split Delivery for Optimized Relief Distribution in Post-Earthquake. Transp. Res. E Logist. Transport. Rev. 2014, 69, 160-179. [CrossRef] 
12. Liu, C.L.; He, J.M.; Sheng, Z.H. Selecting optimal scheme for multiple rescuing emergency systems. J. Ind. Eng./Eng. Manag. 2000, 14, 13-15. (In Chinese)

13. Ben-Tal, A.; Chung, B.D.; Mandala, S.R. Robust Optimization for Emergency Logistic Planning: Risk Mitigation in Humanitarian Relief Supply Chains. Transp. Res. B Meth. 2011, 45, 1177-1189. [CrossRef]

C 2017 by the authors. Licensee MDPI, Basel, Switzerland. This article is an open access article distributed under the terms and conditions of the Creative Commons Attribution (CC BY) license (http:/ / creativecommons.org/licenses/by/4.0/). 